\title{
Acceleration of membrane dynamics adjacent to a wall
}

\author{
H. Frielinghaus, ${ }^{1}$ M. Kerscher,${ }^{2}$ O. Holderer, ${ }^{1}$ M. Monkenbusch, ${ }^{2}$ and D. Richter ${ }^{1,2}$ \\ ${ }^{1}$ Jülich Centre of Neutron Science, Forschungszentrum Jülich GmbH, Lichtenbergstrasse 1, D-85747 Garching, Germany \\ ${ }^{2}$ Institute for Complex Systems 1, Forschungszentrum Jülich GmbH, D-52425 Jülich, Germany
}

(Received 19 August 2011; published 30 April 2012)

\begin{abstract}
The dynamics of an induced lamellar microemulsion adjacent to a planar hydrophilic surface (45 ns) were found to be three times faster compared to the bicontinuous bulk structure (133 ns). For these investigations the grazing incidence technique for neutron spin echo spectroscopy has been developed to resolve the depth dependent near surface dynamics. The observation is rationalized in terms of membrane hydrodynamics, where the flow fields reflected by the surface lead to a crossover from classical to confined fluctuations, and faster dynamics on large length scales (also known as "lubrication") are predicted.
\end{abstract}

DOI: 10.1103/PhysRevE.85.041408

PACS number(s): 82.70.Kj, 64.60.Cn, 68.03.Kn, 68.08.De

\section{INTRODUCTION}

For soft matter systems, confinement [1,2] may be imposed by soft or hard environments. Linear chains in the bulk are often slowed down by the presence of surfaces [3-5]. For glass forming liquids a soft matrix leads to faster relaxation processes [6]. Supercooled water in micelles can be divided into an immobilized surface layer and less confined core water [7]. Cylindrical polymer micelles in a mesophase show enhanced segmental dynamics [8]. Hard confinement of polymers was studied in nanopores, where large scale dynamics were found to be enhanced density dilution [9]. Similar to the water dynamics in micelles, in narrow pores, immobilized shell and liquid core zones were identified for polymers $[3,4]$.

Experimentally, grazing incidence scattering techniques have been employed for measurements of dynamics adjacent to planar surfaces [10-13]. The diffusion of micelles adjacent to a hard wall has been characterized in commissioning experiments [14] using grazing incidence neutron spin echo spectroscopy, but without varying depth resolution. Undulations of membrane stacks in the normal direction have been characterized before $[15,16]$, but in the volume without surface sensitivity.

Surfactant systems and microemulsions appear in enhanced oil recovery [17], where the viscosity and surface tension of a supporting fluid need to be matched to the application. In this application many surfaces exist due to floating particles and the oil-hosting sandstone and thus give rise to confinement effects. Local structures near the surface and orientational ordering with respect to flow [18] may sensitively influence the friction conditions (or lubrication) and the viscosity and therefore the macroscopic pressure needed to inject the fluid.

The static structure of a bicontinuous microemulsion (for an introduction to microemulsions, see $[19,20]$ ) adjacent to a planar hydrophilic surface has been characterized by reflectometry and grazing incidence small angle neutron scattering (GISANS) [21]. In order to study the dynamics of the surface induced structure we have developed the method for grazing incidence neutron spin echo spectroscopy (GINSES). Instead of a reduced dynamics compared to the bicontinuous phase that is observed in the bulk, we observed a threefold acceleration of the membrane dynamics close to the surface (from 133 to $45 \mathrm{~ns}$ ). Following an idea of Seifert [22,23] the acceleration could be semiquantitatively explained in terms of hydrodynamics effects that are imposed by the hard surface.

\section{EXPERIMENTAL DETAIL AND PREVIOUS RESULTS}

GINSES (GISANS) experiments have been performed on J-NSE [24,25] (KWS-2) at the research reactor FRMII in Garching. The wavelength was 0.8 (1.2) $\mathrm{nm}$. For a grazing incidence experiment, we collimated the beam with a slit geometry (see Appendixes A and B). The samples consist of the nonionic surfactant $\mathrm{C}_{10} \mathrm{E}_{4}$ (tetraethylene glycol monodecyl ether at $17 \%$ volume) and equal amounts of oil and water. Isotope mixtures yielded the desired scattering depths (discussed below). Bulk measurements have been performed in transmission geometry [26,27].

A sketch of the scattering geometry is given in Fig. 1. Small incident angles $\alpha_{\mathrm{i}}$ below the critical angle $\alpha_{\mathrm{c}}=\lambda \sqrt{\Delta \rho / \pi}$ cause an evanescent wave with a penetration depth $\Lambda_{\mathrm{i}}=$ $\left[\operatorname{Re} \sqrt{4 \pi \Delta \rho\left(1-\alpha_{i}^{2} / \alpha_{c}^{2}\right)}\right]^{-1}$. So the intensity in the microemulsion highlights a layer of finite thickness. $\Delta \rho$ is the excess scattering length density of the overall microemulsion with respect to the silicon. The scattering depth $\Lambda$ arises from the grazing incidence geometry and reads $\Lambda=\left(\Lambda_{\mathrm{i}}^{-1}+\Lambda_{\mathrm{f}}^{-1}\right)^{-1}$ for the incident and finite wave. Because the exit angle $\alpha_{\mathrm{f}}$ is large $\left(\sim 5.8^{\circ}\right), \Lambda_{\mathrm{f}}$ is virtually infinitely large, and $\Lambda \approx \Lambda_{\mathrm{i}}$. The detailed values for the considered samples are given in Table I. At $\left(Q_{z}, Q_{y}\right) \approx\left(0.5 \mathrm{~nm}^{-1}, 0\right)$ a peak is obtained from the surface induced lamellar order. At $\left(Q_{z}^{2}+Q_{y}^{2}\right)^{1 / 2} \approx$ $0.5 \mathrm{~nm}^{-1}$ a Debye-Scherrer ring appears from the bicontinuous bulk phase. From previous GISANS measurements a relative intensity of the bicontinuous bulk structure with respect to the near surface lamellar structure was obtained as

$$
I_{\text {bic }} / I_{\text {lam }}=6.2 \times 10^{-2}(\Lambda / \mathrm{nm}-42.6) .
$$

For smaller penetration lengths (less than $42.6 \mathrm{~nm}$ ) the lamellar phase dominates the intensity, and so the ratio is virtually zero. The dynamics of the microemulsion adjacent to the wall were measured in $Q$ space, indicated by the red circle in Fig. 1 , so $\left(Q_{z}, Q_{y}\right)=\left(0.8 \mathrm{~nm}^{-1}, 0\right)$. Here, the dynamics of the membrane undulations of the lamellar and bicontinuous phases can be measured as a superposition. The ideal limit for measuring isolated membranes is reached for the highest $Q$, while lower $Q$ values closer to the correlation maximum provide higher intensities. The selected $Q$ avoids de Gennes's narrowing [26,28] but still gives rise to some intermembrane correlations. The limit of detectable signals enforced this choice nonetheless. 


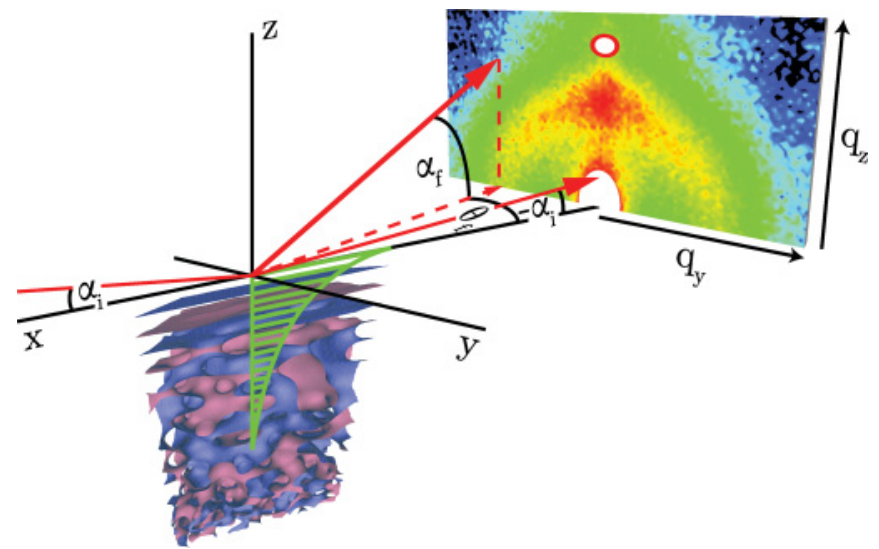

FIG. 1. (Color) Principle of scattering geometry for a GINSES and GISANS experiment. The incidence angle $\alpha_{\mathrm{i}}<\alpha_{\mathrm{c}}$ is extremely small, and the contribution to the scattering angle $\vec{Q} \approx$ $(2 \pi / \lambda)\left(\frac{1}{2} \alpha_{\mathrm{f}}^{2},-\theta_{\mathrm{f}}, \alpha_{\mathrm{f}}\right)$ is negligible. The evanescent wave in the microemulsion (from Ref. [21]) highlights a layer of variable thickness $\Lambda$. The observed scattering vector for the GINSES experiments is indicated by the open red circle.

\section{RESULTS}

Neutron Spin Echo (NSE) measures the intermediate scattering function $S(Q, \tau) / S(Q, 0)$ [24,25], which is shown in Fig. 2 for different scattering depths. The bulk measurement corresponds to an infinitely large scattering depth, and so it has the best statistics. The experiment for $\Lambda=98.5 \mathrm{~nm}$ was improved at $\Lambda=45.3 \mathrm{~nm}$ by using a better polished silicon block. Data for $\Lambda=98.5 \mathrm{~nm}$ fluctuate more than the statistical error bars. This is not attributed to physical effects of the sample but to the instability of the setup. Experimentally, the scattered intensity was increased by a factor of $\sim 3-4$ just by reducing the rms roughness from 0.5 to $0.2 \mathrm{~nm}$. All intermediate scattering functions were described by a stretched exponential function:

$$
\frac{S\left(Q_{z}, \tau\right)}{S\left(Q_{z}, 0\right)}=\exp \left[-\left(\frac{\tau}{\tau_{R}}\right)^{\beta}\right]
$$

All experimental data are described in terms of the relaxation time $\tau_{R}\left(Q_{z}\right)$ from Eq. (2) with a fitted relaxation rate and stretching exponent $\beta$. The relaxation time of the

TABLE I. Sample conditions for the desired scattering depths $\Lambda$. Isotope mixtures in the oil phase $(88 / 12 h$-decane $/ d$-decane) or water phase $\left(91.6 / 8.4 \mathrm{D}_{2} \mathrm{O} / \mathrm{H}_{2} \mathrm{O}\right)$ with the other phases being pure ( $h$-decane or $\mathrm{D}_{2} \mathrm{O}$ ) and assuming the near surface surfactant content being $11 \%$ yielded the correct $\Lambda$. The scattering length densities of the microemulsion $\rho_{\mu \mathrm{E}}$ have to be compared with the silicon $\rho_{\mathrm{Si}}=2.07 \times 10^{-4} \mathrm{~nm}^{-2}$.

\begin{tabular}{llllll}
\hline \hline & \multicolumn{5}{c}{$\Lambda(\mathrm{nm})$} \\
\cline { 2 - 6 } & 45.3 & 48.4 & 60.0 & 98.5 & $\infty$ \\
\hline Isotope mix in & oil & none & none & water & none \\
$\rho_{\mu \mathrm{E}}\left(10^{-4} \mathrm{~nm}^{-2}\right)$ & 2.97 & 2.63 & 2.63 & 2.38 & \\
$\alpha_{\mathrm{c}}(\mathrm{deg})$ & 0.246 & 0.193 & 0.193 & 0.145 & \\
$\alpha_{\mathrm{i}}(\mathrm{deg})$ & 0.19 & 0.12 & 0.15 & 0.12 & \\
\hline \hline
\end{tabular}

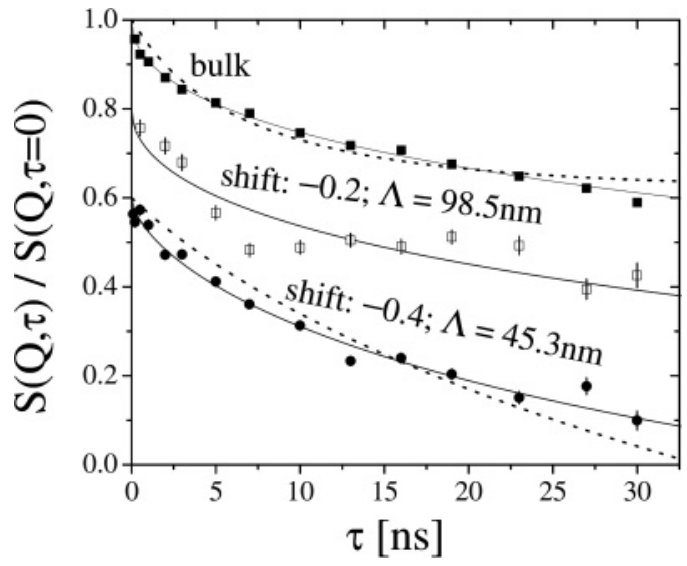

FIG. 2. Intermediate scattering functions of the transmission and grazing incidence NSE measurements $\left(Q=0.08 \mathrm{~nm}^{-1}\right)$. For scattering depths $\Lambda=98.5$ and $45.3 \mathrm{~nm}$ the curves are shifted down by 0.2 and 0.4 (more scattering depths are given in Appendix C). Theoretical descriptions arise from the single stretched exponential [Eq. (2), solid line] and from Zilman-Granek theory with Seifert dispersion relation (dotted line; see text).

bicontinuous structure ( $\tau_{\text {bic }}=133 \pm 3 \mathrm{~ns}$ ) is obtained by a bulk transmission measurement while the characteristic time of the surface near lamellar state $\tau_{\text {lam }}$ was found to be $45 \pm 5 \mathrm{~ns}$. At intermediate $\Lambda$, the measured $\tau_{R}$ (Fig. 3) can be described by a simple superposition of both phases weighted by the intensity contribution obtained from the GISANS experiment:

$$
\tau_{R}=\frac{1}{1+I_{\text {bic }} / I_{\text {lam }}} \tau_{\text {lam }}+\frac{1}{1+I_{\text {lam }} / I_{\text {bic }}} \tau_{\text {bic }} .
$$

A superposition rule for simple exponential decays was applied, although stretched exponential decays were observed. Exponents for the bicontinuous bulk phase and the near surface structure are close to $\frac{1}{2}$ and $\frac{2}{3}$ (Table II).

\section{ANALYSIS AND DISCUSSION}

The stretched exponential function is motivated by the theory of Zilman and Granek [29,30] (Appendix D). They

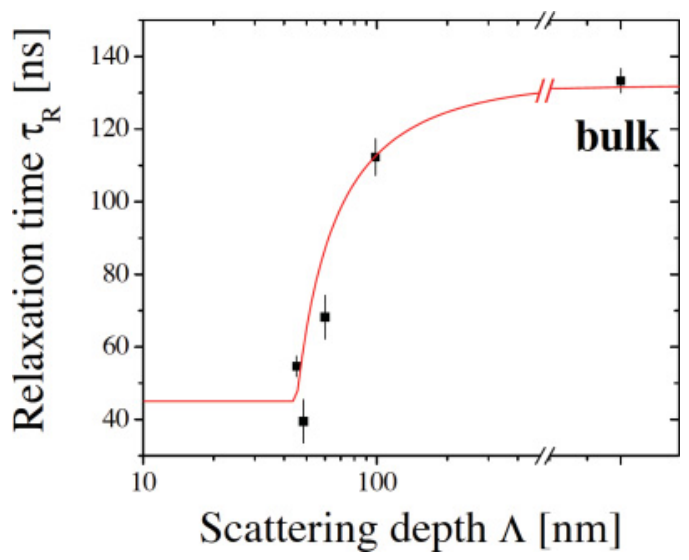

FIG. 3. (Color online) Relaxation times (Kohlrausch-WilliamWatts) obtained from a single stretched exponential fit [Eq. (2)] as a function of the scattering depth $\Lambda$ (symbols). The solid line arises from inserting Eq. (1) in Eq. (3). 
TABLE II. Stretching exponents from a single stretched exponential fit [Eq. (2)] as a function of the scattering depth $\Lambda$.

\begin{tabular}{cccccc}
\hline \hline & \multicolumn{5}{c}{$\Lambda(\mathrm{nm})$} \\
\cline { 2 - 6 } & 45.3 & 48.4 & 60.0 & 98.5 & $\infty$ \\
\hline $100 \beta$ & $63 \pm 2$ & $90 \pm 11$ & $67 \pm 3$ & $49 \pm 5$ & $48 \pm 1$ \\
\hline \hline
\end{tabular}

derived the functional form for large $Q_{z}$ with a stretching exponent of $\beta=\frac{2}{3}$ and a relaxation time $\tau_{R} \propto Q_{z}^{-3}$. A description which allows quantitative determination of the bending rigidity $\kappa$ for isolated soft membranes (at large $Q)$ with $\kappa \sim k_{B} T$ requires numerical integration instead of approximations, leading to Eq. (2):

$$
\begin{aligned}
& S(\vec{Q}, \tau) \\
& \propto\left\langle\int d^{2} r \int d^{2} r^{\prime} \exp \left[i \vec{Q}_{x y}\left(\vec{r}-\vec{r}^{\prime}\right)\right]\right. \\
& \left.\quad \times \exp \left(-\frac{k_{B} T}{4 \pi^{2} \kappa} Q_{z}^{2} \int_{k_{\min }}^{k_{\max }} \frac{d^{2} k}{k^{4}}\left[1-e^{i \vec{k}\left(\vec{r}-\vec{r}^{\prime}\right)-\omega(k) \tau}\right]\right)\right\rangle_{\alpha}
\end{aligned}
$$

The Zilman-Granek theory has been applied successfully in many cases for bulk phases of bicontinuous and lamellar microemulsions [29,31,32]. The angular averaging over the angle $\alpha$ for a bicontinuous microemulsion has to be omitted for the lamellar phase. The bending rigidity $\kappa$ is then the only fitting parameter in this equation for bulk microemulsions. The dispersion relation $\omega(k)=\kappa k^{3} /(4 \eta)$ describes hydrodynamic interactions of the membrane with itself in large volumes (the undulation wave vector $\vec{k}$, which is not to be confused with the scattering vector $\vec{Q}$ ). Integration limits of the patch size $\xi\left(k_{\min }=\pi / \xi\right)$ and the molecule size $a\left(k_{\max }=\pi / a\right.$, with $a \sim 1 \mathrm{~nm}$ being practically infinitesimally small) describe the membrane fluctuations within the patch, while cross correlations between different patches are nonexistent. The limited patch size is physically motivated but also prevents divergences of the inner integral from the $k^{-4}$ term. A more detailed description of the intermediate scattering function can be found in Appendix D. Center of mass diffusion usually is neglected in the nanosecond time scales, especially now for the surface near microemulsion.

The approach of Seifert includes the hydrodynamic and Helfrich interactions of the membrane with the wall (Eq. (10) from Ref. [22]). The first interaction can also be understood as a volume conservation law. A deviating dispersion relation on large length scales $\omega(k) \sim k^{2}$ is obtained. Another detail of the theory is the interaction potential $[23,33]$ describing repulsive (steric) interactions between the membrane segments and the wall and attractive interactions caused by the osmotic pressure of the bulk microemulsion. The potential introduced another length scale $\xi_{v}$ connected to its strength. It has been shown [33] that $\xi_{v}$ is tightly connected to the wall distance $\bar{l}$ via $\xi_{v}=\sqrt{\kappa / k_{B} T} \bar{l}$. The resulting dispersion relation is depicted in Fig. 4. While at high $k$ the free membrane relaxation undulation dispersion $\propto k^{3}$ is retained, at low $k$ the conservation law causes deviations $\propto k^{2}$. Only a very small intermediate $k$ range around $\bar{l}^{-1}$ describes slower fluctuations. The principal results were obtained in parallel in Ref. [34]. To include

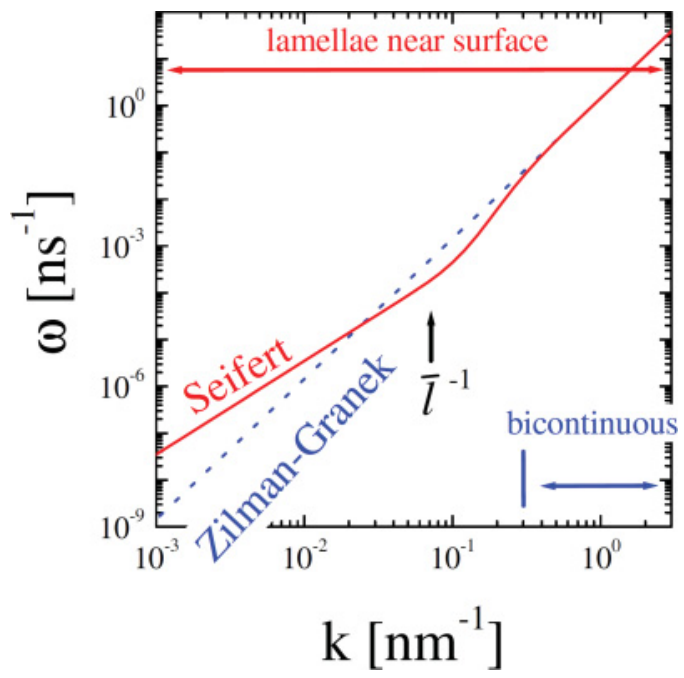

FIG. 4. (Color online) Dispersion relation of the Seifert theory in comparison to the Zilman-Granek theory. The classical ZilmanGranek theory limited the integration $k$ range by the patch size $\xi$, which is eliminated for the Seifert theory. The additional long wavelength modes are responsible for effectively faster relaxations of the lamellar structure close to the surface. The membrane-wall distance is denoted by $\bar{l}$.

the new dispersion relation with the existing Zilman-Granek model the fluctuation amplitudes had to be modified by $k^{-4} \rightarrow\left(k^{4}+\xi_{v}^{-4}\right)^{-1}$, and the integration limits were relaxed to range from zero to infinity. It shall be stressed that basically all fluctuation modes contribute to the intermediate scattering function $S(Q, \tau)$. The low wave numbers obtain a larger weight due to the larger amplitudes, which finally explains our finding of three times faster relaxation rates.

The bulk data from Fig. 2 were fitted with this modified version of the Zilman-Granek theory, with the bending rigidity $\kappa$ and the correlation length $\xi$ as fitted parameters and $\bar{l}=$ $1000 \mathrm{~nm}$ (the membrane is far away from the interface). The bulk data resulted in $\kappa=1.4 k_{B} T$ and $\xi=9.2 \mathrm{~nm}$, i.e., the membrane-membrane distance, as already observed for bulk microemulsions [26]. The data from the experiment with the lowest penetration depth were then fitted with this value of $\kappa$ and $k_{\min }=3 \times 10^{-3} \mathrm{~nm}^{-1}$, which is practically zero. The next three membranes from the interface have been included at a distance $\bar{l}_{1}=2 \bar{l}$, with a weight accounting for lower intensity according to the evanescent wave with a scattering depth $\Lambda$. The parameter $\bar{l}$, describing the distance of the membrane to the wall, has been fitted and found to be $\bar{l}=7.3 \pm 0.5 \mathrm{~nm}$. This analysis shows that the flat wall modifies the spectrum of membrane fluctuations and results in faster relaxations. The parameter $\bar{l}$ has to be compared with the single domain size of $\sim 10 \mathrm{~nm}$, and the idealized first domain size is half this value. For intensity reasons we left the high $Q$ limit of isolated membranes, and so some intermembrane correlations were included in the measurement, which is the reason why the applied theory does not meet the data points well (Fig. 2). Currently, no theory is available to describe interference from different membranes. However, the theory also predicts an acceleration of the relaxations.

The discussion in Ref. [34] displays how the dispersion relation (Fig. 4) is connected to viscosity. The viscosity ratio 
between the induced lamellar order and the bicontinuous microemulsion is given by the ratio of the reciprocal frequencies. At small wave vectors the induced lamellar order facilitates the flow, which is also known as "lubrication," while at large wave vectors there is basically no change. At intermediate wave vectors $\left(k \sim \bar{l}^{-1}\right)$ near surface structure slightly more viscous than for our parameters. Reference [34] considers parameters where the dispersion relation is dramatically slower over a large $k$ range, which is discussed as a high maximal value of the effective viscosity. For classical microemulsions these parameters are unrealistic because the bending rigidity $\kappa / k_{B} T \approx 1 \ll 10$ is much smaller compared to biological membranes. This overall lubrication is highly important for the flow inside porous materials. Macroscopically, it might describe an effective slip (with a finite slip length). From bulk measurements (Appendix E) we conclude that without the confinement effects of the solid wall no acceleration effect and, to some extent, no lubrication effect would be obtained.

The overall physical picture might also be fundamental for understanding the capture process of immune cells (for instance, leukocytes) at vessel walls [35] (or the immune system in general). It has been shown that with diluted receptors (selectins and PNAd) the dissociation times range from $\sim 0.1$ to $1 \mathrm{~s}$ [36]. Even though time reversal cannot be strictly applied within the capture process there must be a surface bound state of the leukocyte before receptors strengthen the surface binding [37]. The confinement of the cell membrane might explain this initial bound state. The cell plasma with all its contents is strongly fluctuating and exerts an osmotic pressure on the surface. The independent patches fluctuate similarly and cause the osmotic pressure on the surface near the lamellae. Interactions repulsive to the vessel wall and our hard model wall should also be comparable. The result is fast fluctuations of the confined membrane with a higher degree of order and a shorter distance than the residual membrane-membrane distances. While the repulsive interactions per unit area are less favorable for smaller $\kappa$ the ability to cover larger wall areas [37] finally leads to stronger binding with smaller $\kappa$. The overall picture can also be seen in context of the Casimir effect [38], which describes attractive forces between metal plates in a fluctuating electromagnetic field of the vacuum. The two half spaces with fluctuations correspond to the fluctuating bulk structure. The restricted electromagnetic waves within the plates correspond to the confined fluctuating cell membrane. The supposed weak attractive interaction of the leukocyte to the vessel wall might cover the needed time for the receptor binding.

\section{SUMMARY}

In summary, the dynamics of a microemulsion were determined as a function of the scattering depth, i.e., as a function of the distance from the confining wall. The Seifert theory for confined membranes applied within the Zilman-Granek theory could semiquantitatively describe the observations.

\section{ACKNOWLEDGMENT}

We thank G. Gompper for discussions.

\section{APPENDIX A: EXPERIMENTAL DETAILS}

GINSES (GISANS) experiments have been performed on J-NSE [24,25] (KWS-2) at the research reactor FRM-II in Garching. The wavelength was 0.8 (1.2) $\mathrm{nm}$. For the grazing incidence geometry the incidence angle needed to be highly defined $\left( \pm 0.02^{\circ}\right)$, which compared to standard experiments reduced the entrance aperture by a factor 30 (from 60 to $2 \mathrm{~mm}$ at a distance of $3.26 \mathrm{~m}$ ). Furthermore, the irradiated sample volume changed from transmission experiments [26,27] $\left(30 \times 30 \times 2 \mathrm{~mm}^{3}\right)$ to a near silicon surface volume of $\sim 20 \times 120 \mathrm{~mm}^{2} \times 40 \mathrm{~nm}$, i.e., by a factor of 19,000. The exceptionally low instrumental background made the experiment nonetheless possible. Typical count rates were found in the range of 4-12 counts/s with a background noise of $2-3$ counts/s. The temperature was $27^{\circ} \mathrm{C}$, which is the phase inversion temperature (of the fishtail point), being $2 \mathrm{~K}$ lower than for fully protonated material. The $2 \mathrm{~cm}$ thick silicon was a monocrystal that was hydrophobically modified [21]. The crystal structure of the silicon is invisible for the neutrons of the considered wavelengths. So the silicon appears as a homogenous material. The bulk measurement has been performed in transmission geometry. The background treatment and standard analysis of NSE measurements was described in Ref. [24].

The viscosities of the reference system with $\mathrm{C}_{10} \mathrm{E}_{4}, \mathrm{D}_{2} \mathrm{O}$, and $h$-decane has only slightly been modified by the isotope exchange. The isotopically controverse materials caused viscosity changes of $-24 \%$ and $+6 \%$. Regarding the relative amounts of isotope exchanges, the corrections were about $\pm 1 \%-2 \%$ which is much less than the errors of the experiment.

The possible treatment of diffusion as an additive term was discussed by Hellweg and coworkers $[39,40]$. The relative amplitudes must favor the undulations at high $Q$. Unfortunately, no typical relative amplitudes are published for medium $Q$, and so we refrained from taking diffusion into account.

\section{APPENDIX B: INTENSITY CONSIDERATIONS}

Typical film contrast samples as a conventional bulk sample show count rates of typically 5000 counts/s. The bulk contrast leads to a gain factor of $\sim 20$. For the near surface structure in a GINSES experiment below the critical angle another gain factor of up to 4 is expected. The lamellar structure coherently superimposes single lamellae scattering contributions (gain $\sim 16$ ). These gains result in a count rate of $\sim 6 \times 10^{6}$ counts $/ \mathrm{s}$ which are reduced by the geometrical factors of 19000 and 30 down to $\sim 11$ counts/s. At small enough length scales ( $Q>Q_{\max }$, given by the correlation peak) the normalized intermediate scattering functions of bulk and film contrast depict the same dynamics and therefore have identical forms [28].

\section{APPENDIX C: COMPLETE RELAXATION CURVES}

While we could only present some representative relaxation curves in the main paper (see Fig. 2) the full data set is displayed here (Fig. 5). The intermediate penetration depths $\Lambda=48.4, \ldots, 98.5 \mathrm{~nm}$ have historically been obtained from the less well polished silicons, and so the noise of the measurements were distinguishably higher than for the well polished silicon block $(\Lambda=45.3 \mathrm{~nm})$. Nonetheless, the data 


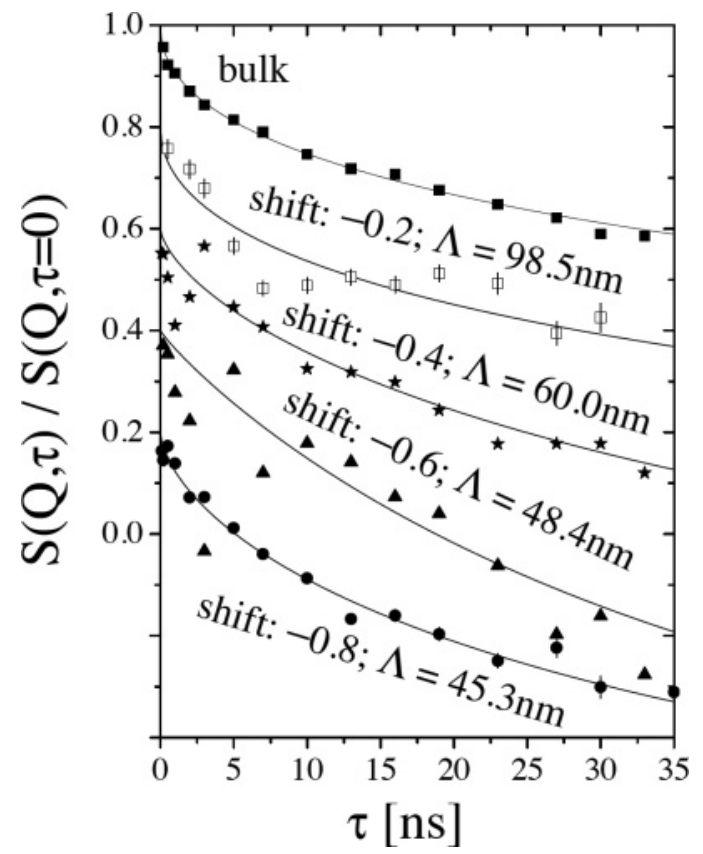

FIG. 5. Full set of intermediate scattering functions of the transmission and grazing incidence NSE measurements $\left(Q=0.08 \mathrm{~nm}^{-1}\right)$. The single curves for different scattering depths $\Lambda$ are sequentially shifted down as indicated. The solid lines arise from a single stretched exponential fit [Eq. (2)].

show a clearly measured effect, and all realistic error bars are given in the main paper.

\section{APPENDIX D: INTERMEDIATE SCATTERING FUNCTION OF A MEMBRANE PATCH}

Neutron spin echo spectroscopy measures the intermediate scattering function $S(Q, \tau)$. The dynamic structure factor of a membrane patch as the basic constituent of microemulsions is $[29,30]$

$$
\begin{aligned}
S(\vec{Q}, \tau) \propto & \left\langle\left\langle\int_{\text {patch }} d^{2} r \int_{\text {patch }} d^{2} r^{\prime} \exp \left[i \vec{Q}_{\|}\left(\vec{r}-\vec{r}^{\prime}\right)\right]\right.\right. \\
& \left.\times \exp \left\{i Q_{z}\left[h(\vec{r}, \tau)-h\left(\vec{r}^{\prime}, 0\right)\right]\right\}\right\rangle\left.\right|_{\alpha} .
\end{aligned}
$$

After statistical averaging over the last exponential factor, one gets

$$
\begin{aligned}
S(\vec{Q}, \tau) \propto & \left\langle\int_{\text {patch }} d^{2} r \int_{\text {patch }} d^{2} r^{\prime} \exp \left[i \vec{Q}_{\|}\left(\vec{r}-\vec{r}^{\prime}\right)\right]\right. \\
& \left.\times \exp \left(-Q_{z}^{2}\left\langle\left[h(\vec{r}, \tau)-h\left(\vec{r}^{\prime}, 0\right)\right]^{2}\right\rangle\right)\right\rangle_{\alpha}
\end{aligned}
$$

The angular averaging with angle $\alpha$ is needed for bicontinuous microemulsions with their statistically distributed directions of the surface normal and is omitted for oriented lamellar microemulsions $[26,31,32]$.

The function $h(\vec{r}, \tau)$ describes the membrane displacement from its average position. The exponent in Eq. (D1) with Fourier components $h_{\vec{k}}$ with wave vector $\vec{k}$ of the undulations can be expressed by

$$
\left\langle h_{\vec{k}}(\tau) h_{-\vec{k}}(0)\right\rangle=\frac{k_{B} T}{\kappa k^{4}} \exp [-\omega(k) \tau] .
$$

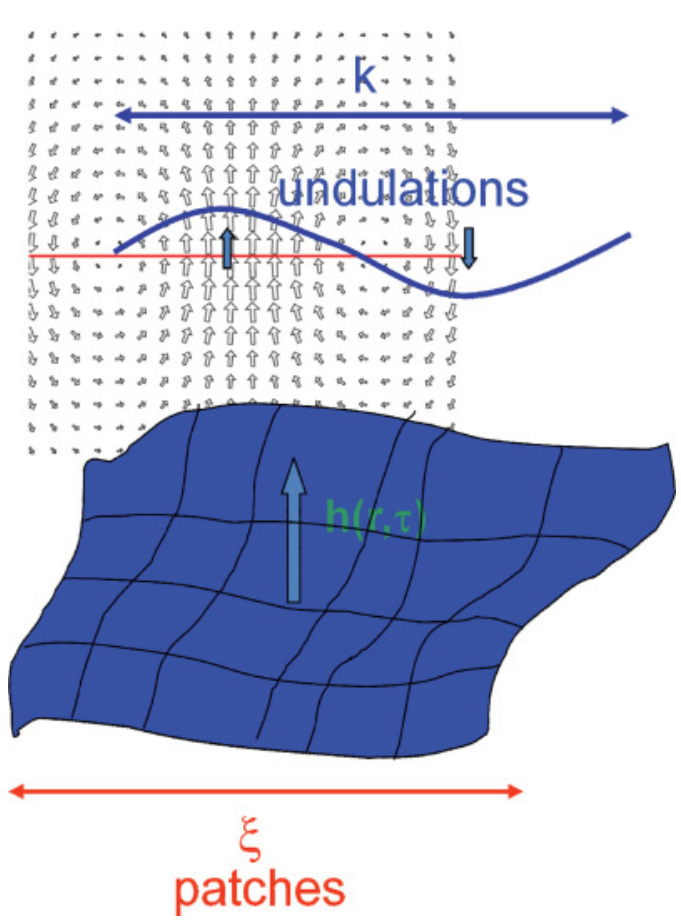

FIG. 6. (Color online) Illustration of the Zilman-Granek model of a fluctuating membrane patch surrounded by a viscous medium. The wave vector $\vec{k}$ lies in the plane of the membrane, and the displacement from the equilibrium position is described by $h(\vec{r}, \tau)$.

The dispersion relation for bulk microemulsions is thereby $\omega(k)=\kappa k^{3} /(4 \eta)$. After some mathematics one arrives at

$$
\begin{aligned}
& S(\vec{Q}, \tau) \\
& \propto\left\langle\int_{\text {patch }} d^{2} r \int_{\text {patch }} d^{2} r^{\prime} \exp \left[i \vec{Q}_{\|}\left(\vec{r}-\vec{r}^{\prime}\right)\right]\right. \\
& \left.\quad \times \exp \left(-\frac{k_{B} T}{4 \pi^{2} \kappa} Q_{z}^{2} \int_{k_{\min }}^{k_{\max }} \frac{d^{2} k}{k^{4}}\left[1-e^{i \vec{k}\left(\vec{r}-\vec{r}^{\prime}\right)-\omega(k) \tau}\right]\right)\right\rangle_{\alpha} .
\end{aligned}
$$

A detailed derivation of this formula can be found in Refs. [22, 32] (Fig. 6). Thermally induced undulations are limited to wave vectors $\vec{k}$, which on the side of large wave vectors are limited by the surfactant molecule size which constitutes the membrane, i.e., $k_{\max }<\pi / a$, because undulations with a wavelength shorter than the surfactant molecule are not possible. On the other hand, the long wavelength limit (i.e., small- $k$ limit) is of the order of the patch size of a membrane, $k_{\min }>$ $\pi / \xi$. Undulation modes are limited to wavelengths shorter than $\xi$; the correlation length serves as a cutoff parameter of the undulation mode spectrum for bulk microemulsions. Experiments on bicontinuous microemulsions showed the validity of this assumption of a long wavelength cutoff [26,31] with $k_{\min }>1.26 \times \pi / \xi$.

We want to emphasize that the experimental scattering vector $Q$ is not identical to the undulation wave vector $k$, but just defines the length scale on which one experimentally probes the spectrum of the membrane undulations. The correlation length $\xi$ is a measure of the length scale, on which one can predict the orientation of a membrane. 


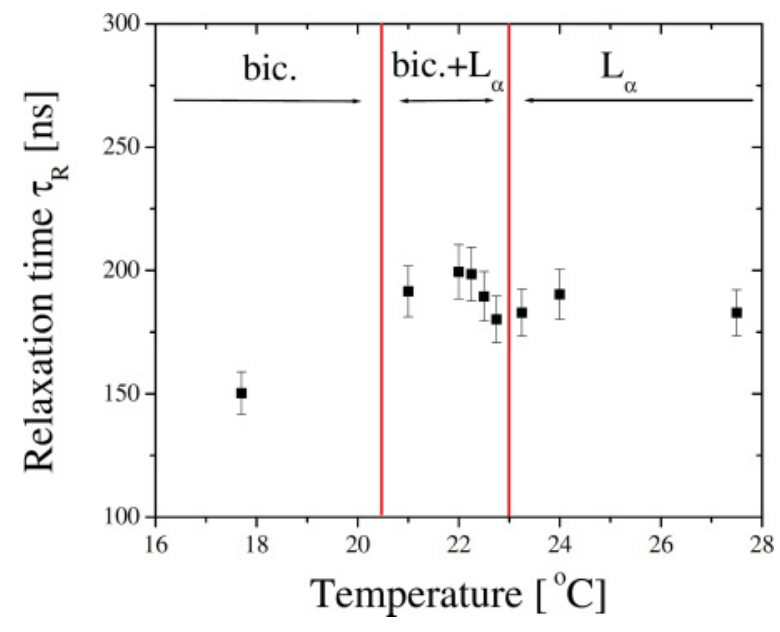

FIG. 7. (Color online) Relaxation rates of transmission measurements for higher surfactant concentrations $\gamma=32.2 \%$. For different temperatures different phases are entered, i.e., the bicontinuous (bic.) and lamellar $\left(L_{\alpha}\right)$ phases and a coexistence region. The obtained relaxation times are grossly independent of the structure.

A membrane close to a flat wall experiences additional contributions to the membrane energy which have been formulated by Seifert [22,23]. The dispersion relation for small wave vectors $k$ is proportional to $k^{2}$, as shown in Fig. 4. We exchanged the dispersion relation in Eq. (D4) with the expression from Seifert [Eqs. (9), (10), and (16) in Ref. [33], where the distance to the flat wall $\bar{l}$ determines how the undulation mode spectrum is modified:

$$
\omega\left(k, \bar{l}, \xi_{v}\right)=\Gamma(k, \bar{l}) E\left(k, \xi_{v}\right),
$$

with

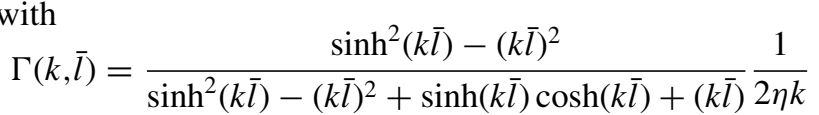

and

$$
E\left(k, \xi_{v}\right)=\kappa\left(k^{4}+\xi_{v}^{-4}\right)+\Sigma k^{2} .
$$

The surface tension $\Sigma$ in Eq. (D7) has been set to zero. The ideal correlation length in the Seifert model is $\xi_{v}=\sqrt{\kappa / k_{B} T} \bar{l}$ [33]. Putting this dispersion relation into Eq. (D4) and fitting the GINSES data with this modified theory allow determining $\bar{l}$. The relaxation time is modified by the changes of the undulation mode spectrum due to the wall-membrane interaction. For membrane patches far away from the membrane, $\bar{l}$ is large and the bulk dispersion relation is recovered (see Fig. 4).

\section{APPENDIX E: COMPARISON OF BULK BICONTINUOUS AND LAMELLAR PHASES}

The considerable changes in the relaxation rates cannot be explained on the basis of the membrane morphology alone. Bulk measurements of coexisting lamellar and bicontinuous phases were compared experimentally (Fig. 7). While the pure bicontinuous microemulsion shows a relaxation time of $150 \mathrm{~ns}$, the two coexisting phases as well as the pure lamellar phase indicate relaxation times of $\sim 190 \mathrm{~ns}$. Thus in the bulk the lamellar phase relaxes more slowly than the bicontinuous phase.

The whole experiment has to be understood in the context of scaling. The higher surfactant concentrations of $32 \%$ lead to smaller domains and thus to a shift to higher $Q$ values. This is seen in the peak position, and the observation $Q$ for NSE measurements has been shifted accordingly. Temperature changes within the bulk experiment are of the order $\pm 2 \%$ and can be safely neglected as effects on the bending rigidity and thus on the relaxation times within the precision of the experiment.
[1] G. B. McKenna, Eur. Phys. J. 189, 285 (2010).

[2] V. G. Sakai and A. Arbe, Curr. Opin. Colloid Interface Sci. 14, 381 (2009).

[3] M. Krutyeva, J. Martín, A. Arbe, J. Colmenero, C. Mijangos, G. J. Schneider, T. Unruh, Y. Su, and D. Richter, J. Chem. Phys. 131, 174901 (2009).

[4] A. Kusmin, S. Gruener, A. Henschel, O. Holderer, J. Allgaier, D. Richter, and P. Huber, J. Phys. Chem. Lett. 1, 3116 (2010).

[5] K. Nusser, S. Neueder, G. J. Schneider, M. Meyer, W. Pyckhout-Hintzen, L. Willner, A. Radulescu, and D. Richter, Macromolecules 43, 9837 (2010).

[6] R. Zorn, M. Mayorova, D. Richter, and B. Frick, Soft Matter 4, 522 (2008).

[7] T. L. Spehr, B. Frick, M. Zamponi, and B. Stühn, Soft Matter 7, 5745 (2011).

[8] L. Willner, R. Lund, M. Monkenbusch, O. Holderer, J. Colmenero, and D. Richter, Soft Matter 6, 1559 (2010).

[9] J. Martín, M. Krutyeva, M. Monkenbusch, A. Arbe, J. Allgaier, A. Radulescu, P. Falus, J. Maiz, C. Mijangos, J. Colmenero, and D. Richter, Phys. Rev. Lett. 104, 197801 (2010).
[10] J. Daillant, Curr. Opin. Colloid Interface Sci. 14, 396 (2009).

[11] A. Madsen, J. Als-Nielsen, and G. Grubel, Phys. Rev. Lett. 90, 085701 (2003).

[12] A. Madsen, T. Seydel, M. Sprung, C. Gutt, M. Tolan, and G. Grübel, Phys. Rev. Lett. 92, 096104 (2004).

[13] B. M. Murphy, H. Requardt, J. Stettner, J. Serrano, M. Krisch, M. Muller, and W. Press, Phys. Rev. Lett. 95, 256104 (2005).

[14] M. Walz, S. Gerth, P. Falus, M. Klimczak, T. H. Metzger, and A. Magerl, J. Phys.: Condens. Matter 23, 324102 (2011).

[15] M. C. Rheinstädter, W. Häußler, and T. Salditt, Phys. Rev. Lett. 97, 048103 (2006).

[16] I. Sikharulidze, B. Farago, I. P. Dolbnya, A. Madsen, and W. H. de Jeu, Phys. Rev. Lett. 91, 165504 (2003).

[17] V. Santanna, F. Curbelo, T. Dantas, A. Neto, H. Albuquerque, and A. Garnica, J. Petrol. Sci. Eng. 66, 117 (2009).

[18] S. Förster, M. Konrad, and P. Lindner, Phys. Rev. Lett. 94, 017803 (2005).

[19] M. Kahlweit, R. Strey, D. Haase, H. Kunieda, T. Schmeling, B. Faulhaber, M. Borkovec, H. F. Eicke, G. Busse, F. Eggers, T. Funck, H. Richmann, L. Magid, O. Södermann, P. Stilbs, 
J. Winkler, A. Dittrich, and W. Jahn, J. Colloid Interface Sci. 118, 436 (1987).

[20] X. L. Zhou, L. T. Lee, S. H. Chen, and R. Strey, Phys. Rev. A 46, 6479 (1992).

[21] M. Kerscher, P. Busch, S. Mattauch, H. Frielinghaus, D. Richter, M. Belushkin, and G. Gompper, Phys. Rev. E 83, 030401 (2011).

[22] M. Kraus and U. Seifert, J. Phys. II 4, 1117 (1994).

[23] U. Seifert, Phys. Rev. E 49, 3124 (1994).

[24] M. Monkenbusch, R. Schätzler, and D. Richter, Nucl. Instrum. Methods Phys. Res., Sect. A 399, 301 (1997).

[25] O. Holderer, M. Monkenbusch, R. Schätzler, H. Kleines, W. Westerhausen, and D. Richter, Meas. Sci. Technol. 19, 034022 (2008).

[26] O. Holderer, H. Frielinghaus, D. Byelov, M. Monkenbusch, J. Allgaier, and D. Richter, J. Chem. Phys. 122, 094908 (2005).

[27] B. Farago and M. Gradzielski, J. Chem. Phys. 114, 10105 (2001).

[28] O. Holderer, H. Frielinghaus, M. Monkenbusch, J. Allgaier, D. Richter, and B. Farago, Eur. Phys. J. E 22, 157 (2007).

[29] A. G. Zilman and R. Granek, Phys. Rev. Lett. 77, 4788 (1996).

[30] A. G. Zilman and R. Granek, Chem. Phys. 284, 195 (2002).
[31] M. Mihailescu, M. Monkenbusch, H. Endo, J. Allgaier, G. Gompper, J. Stellbrink, D. Richter, B. Jakobs, T. Sottmann, and B. Farago, J. Chem. Phys. 115, 9563 (2001).

[32] M. Mihailescu, M. Monkenbusch, J. Allgaier, H. Frielinghaus, D. Richter, B. Jakobs, and T. Sottmann, Phys. Rev. E 66, 041504 (2002).

[33] G. Gompper and D. M. Kroll, Europhys. Lett. 9, 59 (1989).

[34] N. Gov, A. G. Zilman, and S. Safran, Phys. Rev. E 70, 011104 (2004).

[35] K. Ley, C. Laudanna, M. I. Cybulsky, and S. Nourshargh, Nat. Rev. Immunol. 7, 678 (2007).

[36] R. Alon, S. Chen, K. D. Puri, E. B. Finger, and T. A. Springer, J. Cell Biol. 138, 1169 (1997).

[37] G. P. Robbins, D. Lee, J. S. Katz, P. R. Frail, M. J. Therien, J. C. Crocker, and D. A. Hammer, Soft Matter 7, 769 (2011).

[38] I. V. Fialkovsky, V. N. Marachevsky, and D. V. Vassilevich, Phys. Rev. B 84, 035446 (2011).

[39] S. Wellert, M. Karg, O. Holderer, A. Richard, and T. Hellweg, PhysChemChemPhys 13, 3092 (2011).

[40] S. Wellert, B. Tiersch, J. Koetz, A. Richard, A. Lapp, O. Holderer, J. Gäb, M. M. Blum, C. Schulreich, R. Stehle, and T. Hellweg, Eur. Biophys. J. 40, 761 (2011). 